LPS is an essential component of the cell surface of a subset of bacterial pathogens called Gram-negative bacteria.

Using genetically mutated bacteria and purified sugar molecules, the authors sought to pinpoint the molecules in the LPS biosynthetic pathway that stimulate NF- $\kappa$ B activation. They found that the presence of bacterial sugars, including ADP- $\beta$-D-manno-heptose (ADPHep) and D-glycero- $\beta$-D-manno-heptose 1,7-bisphosphate (HBP), in the host-cell cytoplasm triggered NF- $\kappa \mathrm{B}$ activation. This is consistent with a study ${ }^{5}$ of Neisseria meningitidis bacteria that demonstrated that $\mathrm{HBP}$ can trigger NF- $\kappa \mathrm{B}$ responses in host cells. Crucially, Zhou et al. showed that ADP-Hep is 100 times more potent than is HBP at activating NF- $\kappa \mathrm{B}$. They found that addition of ADP-Hep to the extracellular environment of host cells can activate NF- $\kappa \mathrm{B}$, suggesting that dedicated host-cell transporter proteins deliver ADP-Hep to the host's cytoplasm.

No PRR was known to recognize ADP-Hep. To search for one, the authors used a geneediting approach to conduct a screen in which they generated random mutations in host cells and tested whether the mutations affected ADP-Hep recognition. They uncovered two candidate genes that respectively encode the kinase enzyme ALPK1 and the protein TIFA, and showed that these are required for NF- $\kappa B$ activation in response to ADP-Hep in host cells (Fig. 1). A previous study had revealed ${ }^{5}$ that TIFA is required for recognition of HBP from N. meningitidis. ALPK1 and TIFA signalling has also been linked to HBP-dependent host activation of NF- $\mathrm{\kappa B}$ in response to infection by the bacteria Shigella flexneri ${ }^{6}$ and Helicobacter pylori ${ }^{7}$. Using biochemical approaches, Zhou and colleagues demonstrated that ADP-Hep binds directly to the amino terminus of ALPK1. The authors solved the X-ray crystal structure of ALPK1 in a complex with ADP-Hep, and validated their structural model by testing the effect of mutations in ALPK1 that were predicted to impair its binding to ADP-Hep.

Zhou et al. also generated ALPK1-deficient mice. The NF- $\kappa \mathrm{B}$-dependent production of cytokines was significantly reduced in these animals after challenge with either ADP-Hep or the pathogenic bacterium Burkholderia cenocepacia, compared with results seen in animals that were not deficient in ALPK1. Moreover, the number of bacteria in the lungs of mice infected with B. cenocepacia was higher in ALPK1deficient animals than in wild-type mice.

Perhaps Zhou and colleagues' most striking finding is that mammalian adenylyltransferase enzymes, specifically those of the NMNAT family, catalyse a reaction that converts HBP into a molecule called ADP-heptose 7-P, which can act as a ligand by binding to ALPK1. Previous work ${ }^{5}$ had suggested that HBP is a PAMP that can directly activate NF- $\mathrm{BB}$. Although $\mathrm{HBP}$ can be defined as a PAMP, given that it is a bacterially derived molecule that triggers a host response, Zhou and colleagues' data indicate that HBP must be converted to ADP-heptose 7-P by host enzymes to trigger this response. The authors report slight differences in the way in which ADP-Hep and ADP-heptose 7-P bind to ALPK1, and use these differences to demonstrate why ADP-Hep and not HBP or ADP-heptose 7-P is the relevant ligand for ALPK1-mediated NF- $\kappa \mathrm{B}$ activation, at least in Y.pseudotuberculosis infection.

Zhou and colleagues' findings have important implications. Evidence that ADP-Hep is a PAMP adds to a growing awareness that bacterial metabolites can act as PAMPs. Given that ADP-Hep is needed to synthesize an essential component of the outer membrane of most Gram-negative bacteria, this makes it an ideal PAMP. However, it is not known how this molecule, which is normally found inside the bacterium, reaches the cytoplasm of the host cell. In Y.pseudotuberculosis, this process requires the T3SS, although it is unclear whether ADP-Hep is actively transported or accidentally leaks through the T3SS, or whether it enters by the pores that the T3SS generates in the host-cell membrane.

The authors report that bacterial species that lack a T3SS can still trigger the ALPK1 pathway in an ADP-Hep-dependent manner, consistent with the ability of purified ADP-Hep to activate the pathway by an extracellular route. This suggests that a dedicated transport system might exist that allows the host cell to sample its extracellular surroundings for the presence of this PAMP, similar to the way in which certain extracellular PAMPs are transported to the cytoplasm for recognition by host proteins ${ }^{8}$.

Why does bacterial ADP-Hep exposure occur if it activates the innate immune system? Perhaps its release is needed to fulfil some as yet unknown function. Pathogens often evolve mechanisms to evade or thwart an immunesystem response. If pathogens have evolved strategies to avoid triggering an ADP-Hepmediated immune response, understanding such strategies might suggest new therapeutic approaches to fight bacterial infections.

The authors' observation that host enzymes can convert bacterial metabolites that have poor immune-activating characteristics into potent PAMPs offers a new perspective on the evolutionary battle between pathogens and their hosts. Although Zhou et al. show that ADP-Hep is the relevant immune-triggering ligand for Y. pseudotuberculosis infections, it remains to be seen whether HBP is converted into ADP-heptose 7-P during other bacterial infections. This issue is particularly relevant for pathogens (for example, Shigella) that invade the host-cell cytoplasm and that might shed PAMPs such as HBP directly into the cytoplasm. Zhou and colleagues' work also offers a fresh perspective on the types of molecule that can act as PAMPs or their PRRs, and where and how researchers should be searching for such molecules.

John-Demian Sauer is in the Department of Medical Microbiology and Immunology, University of Wisconsin-Madison, Madison, Wisconsin 53706, USA.

e-mail:sauer3@wisc.edu

1. Zhou, P. et al. Nature 561, 122-126 (2018)

2. Lemaitre, B., Nicolas, E., Michaut, L., Reichhart, J.-M. \& Hoffmann, J. A. Cell 86, 973-983 (1996).

3. Medzhitov, R., Preston-Hurlburt, P. \& Janeway, C. A. Jr Nature 388, 394-397 (1997).

4. Poltorak, A. et al. Science 282, 2085-2088 (1998).

5. Gaudet, R. G. et al. Science 348, 1251-1255 (2015).

6. Milivojevic, M. et al. PLoS Pathog. 13, e1006224 (2017).

7. Zimmermann, S. et al. Cell Rep. 20, 2384-2395 (2017).

8. Lee, J. et al. J. Biol. Chem. 284, 23818-23829 (2009).

This article was published online on 15 August 2018.

\title{
CHEMICAL BIOLOGY
}

\section{DNA tags light up sugars on proteins}

Methods for imaging sugars attached to proteins - the protein glycoforms - are of interest because glycoforms affect protein movement and localization in cells. A versatile approach is now reported that uses DNA as molecular identity tags.

\section{TADASHI SUZUKI}

$\uparrow$ The attachment of sugar molecules to proteins is one of the most common protein modifications, found in all domains of life. Sugars attached to proteins are called glycans, and modulate the physicochemical and physiological properties of the carrier proteins ${ }^{1}$. But tracking and visualizing glycoforms - the specific patterns of sugars attached to a protein - in cells is challenging, particularly if you want to visualize several different glycoforms at once. Writing in Angewandte Chemie, Li et al. ${ }^{2}$ now report a method for doing this that relies on the dynamic interactions of a set of DNA codes.

Since the early 1990s, the use of fluorescent tags as labels for proteins has revolutionized 
a

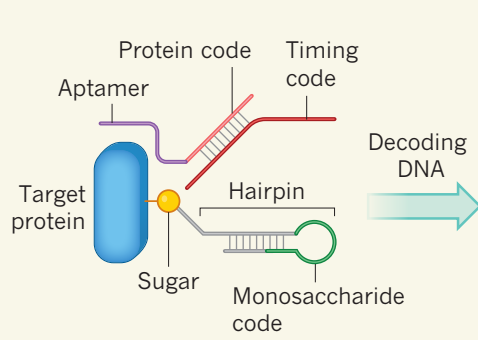

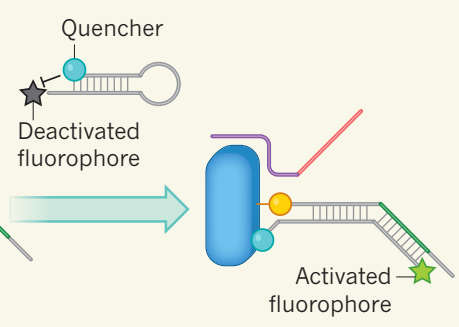

Figure 1 | A method for visualizing sugars on proteins ${ }^{2}$. a, A DNA sequence (the protein code) is bound to the target protein through a sequence called an aptamer. The protein code is hybridized (forms a double helix) with a second sequence, called the timing code. A sugar attached to the protein is covalently attached to a 'hairpin' DNA, which contains a masked sequence that identifies the sugar (the monosaccharide code). b, A 'decoding' DNA molecule is added that hybridizes with the timing code (not shown), releasing the protein code so that it hybridizes with part of the hairpin. The hairpin opens, unmasking the monosaccharide code. c, A second hairpin DNA is added, which is complementary to the monosaccharide code; it also bears a fluorescent molecule (a fluorophore) at one end and a quencher molecule at the other, which deactivates fluorescence. The hairpin hybridizes with the DNA containing the monosaccharide code, opening the hairpin and allowing the fluorophore to fluoresce. The protein code is simultaneously exposed by this process, and can take part in another cycle of reactions.

how cell biologists analyse protein movement and localization in cells $s^{3,4}$. But even though the types of glycan attached to proteins can affect their movement and localization, it has been difficult to visualize any particular glycoform. One way in which researchers have attempted to solve this problem is by using a technique called fluorescence resonance energy transfer (FRET). In this technique, a fluorescent molecule (a fluorophore) is attached to a protein of interest and a second fluorophore is attached to a specific sugar; fluorescence occurs only if the two molecules come into close proximity through the attachment of the sugar to the protein $^{5-8}$. However, the need to use two different fluorophores can limit applications, for example by making it difficult to detect multiple glycoforms of a protein in the same experiment.

Li et al. overcome this problem using an approach that they describe as a hierarchical coding strategy, in which multiple single-stranded DNA molecules are used as identification codes to visualize specific sugars attached to a chosen protein (Fig. 1). The first DNA molecule used in the authors' system contains a sequence (known as an aptamer) that specifically binds to the target protein. The aptamer is attached to another sequence (the protein code) that identifies the protein. A second DNA molecule, called the timing code, contains a sequence that is complementary to the protein code, and that therefore hybridizes (forms a double helix) with it.

The third DNA molecule used in Li and colleagues' system contains three segments. The first segment is complementary to the protein code. This is attached to a second sequence called the monosaccharide code, which identifies a specific sugar. The third segment has a sequence that enables the complete strand to form a structure known as a hairpin, which masks the monosaccharide code. The hairpin DNA is covalently attached to the sugar identified by the monosaccharide code. If the hairpin-bearing sugar is in turn attached to the target protein, this can bring the hairpin into close proximity with the double helix formed by the protein and timing codes.

The final key component of $\mathrm{Li}$ and colleagues' system is another hairpin DNA, which contains a complementary sequence to the monosaccharide code and a sequence that can displace the protein code from a double helix. The hairpin also has a fluorophore attached at the 5' end, and a 'quencher' molecule at the $3^{\prime}$ end. The quencher stops the fluorophore from fluorescing when the hairpin is closed, but allows fluorescence when the hairpin opens.

So how do all these components interact to decode the crucial DNA identifiers and allow glycoforms to be visualized? The process is triggered when a single-stranded DNA that is complementary to the timing code is added to the system. This DNA hybridizes with the timing code, thus displacing and exposing the protein code. The exposed protein code then hybridizes with the complementary sequence in the hairpin attached to the sugar, opening up the hairpin and unmasking the monosaccharide code.

When the fluorophore-carrying hairpin is added to the system, the unmasked monosaccharide code hybridizes with the complementary DNA sequence in that hairpin. The hairpin therefore opens up, allowing its fluorophore to fluoresce: in effect, a fluorescent tag has been attached to the sugar, allowing it to be detected. The hybridization also unmasks the protein code, making it available for another reaction cycle. The key element of $\mathrm{Li}$ and colleagues' system is that the protein code is physically associated with the target protein, because this ensures that only hairpinbearing sugars that are attached or close to the protein can become fluorescent.
The authors confirmed that the chain of reactions occurs in a cell-free system in vitro and used it to identify two glycoforms of the MUC1 protein: MUC1 decorated with the sugar fucose, or with another sugar called sialic acid. Crucially, the authors also showed that the fluorescent signals can be generated and detected on cells that had been modified using a method known as metabolic labelling ${ }^{9}$ to incorporate hairpin-bearing sugars.

An advantage of this method is that, because the choice of DNA sequences that can be used as labels is effectively infinite, many different glycoforms can be imaged, as long as the proteins and sugars can be specifically labelled with their own DNA codes. Moreover, the authors clearly showed that sialylated and fucosylated MUC1 could be simultaneously detected using their method. One potential limitation, however, is that the DNA used was not observed to be transported into cells through natural processes, suggesting that intracellular glycoforms cannot be detected by this method. This could actually be an advantage for studies that focus on cell-surface proteins.

A few issues will need to be clarified in future studies. For example, the efficiency of the decoding process is unclear. It is also not known whether sugars on molecules next to the target proteins might sometimes become fluorescent, as a result of DNA hybridization between the protein code and hairpins attached to sugars on neighbouring proteins. Because a large number of glycans are attached to MUC1, the method might not need to be highly efficient to generate a detectable fluorescent signal for this protein, and any minute signals produced from neighbouring molecules would not be a serious problem. However, further experiments using other glycoproteins that have fewer sugars attached are needed to validate the method fully.

Given that both the above issues might depend largely on the length of the DNA chains used, careful design of the DNA codes and of the aptamers will be essential for ensuring the specific detection of other glycoforms. The practical advantages and disadvantages of the new technique compared with other strategies for glycoform imaging that have been reported in the past few years - including two methods reported by workers from the same group as Li et al..$^{10,11}$ - also remain to be explored.

Nevertheless, Li and colleagues' hierarchical coding strategy for glycoform imaging shows great potential, and could be an important step in the development of a system analogous to the use of green fluorescent proteins for protein tagging - which is now standard practice for biologists. The ultimate goal is to visualize glycoforms in a way that will enable us to see what we want to see, rather than only what can be seen.

Tadashi Suzuki is in the Glycometabolic Biochemistry Laboratory, RIKEN Cluster 
for Pioneering Research, Saitama 351-0198, Japan.

e-mail:tsuzuki_gm@riken.jp

1. Varki, A. Glycobiology 27, 3-49 (2017).

2. Li, S. et al. Angew. Chem. Int. Edn https://doi. org/10.1002/anie.201807054 (2018).
3. Miyawaki, A. Nature Rev. Mol. Cell Biol. 12, 656-668 (2011).

4. Hori, Y. \& Kikuchi, K. Curr. Opin. Chem. Biol. 17, 644-650 (2013)

5. Haga, Y. et al. Nature Commun. 3, 907 (2012).

6. Lin, W., Du, Y., Zhu, Y. \& Chen, X. J. Am. Chem. Soc. 136, 679-687 (2014)

7. Belardi, B. et al. Angew. Chem. Int. Edn $\mathbf{5 2}$ 14045-14049 (2013).
8. Doll, F. et al. Angew. Chem. Int. Edn 55, 2262-2266 (2016).

9. Laughlin, S. T. \& Bertozzi, C. R. Nature Protocols 2, 2930-2944 (2007).

10.Wu, N., Bao, L., Ding, L \& Ju, H. Angew. Chem. Int. Edn 55, 5220-5224 (2016).

11. Hui, J. et al. Angew. Chem. Int. Edn 56, 8139-8143 (2017).

\section{A systemic problem with pesticides}

\begin{abstract}
Exposure to a sulfoximine-based pesticide has substantial adverse effects on bumblebee colonies. This finding suggests that concerns over the risks of exposing bees to insecticides should not be limited to neonicotinoids. SEE LETTER P.109
\end{abstract}

\section{NIGEL E. RAINE}

A gricultural intensification has increased our reliance on pesticides, including insecticides. Although insecticides by insect pests, they can also affect beneficial insects, potentially impairing their ability to control pests and pollinate crops ${ }^{1}$ - qualities on which farmers rely. Indeed, increases in insecticide use are one of several major factors implicated in the worldwide declines of insect pollinators ${ }^{2}$. A commonly used class of insecticide called neonicotinoids has hit the headlines because of its impacts on bees. Siviter et $_{\text {al. }}^{3}$ report on page 109 that a potential neonicotinoid replacement, the sulfoximinebased insecticide sulfoxaflor, also harms these crucial pollinators.

Insect pollinators that forage on neonicotinoid-treated plants can be exposed to small amounts of insecticide each time they or their larvae feed on pollen and nectar ${ }^{4,5}$. Although such chronic neonicotinoid exposure typically does not kill bees, it can have sublethal effects - impairing a range of behaviours such success, colony development and reproduction $^{7-12}$, and reducing pollination levels ${ }^{13}$. Because of this, substantial restrictions on neonicotinoid use have been introduced in some regions of the world, particularly Europe. Such restrictions might seem to be good news for bee health - but only if the insecticides that replace neonicotinoids are less harmful to insect pollinators.

Similar to neonicotinoids, sulfoximine-based insecticides are absorbed and systemically distributed throughout the plant. Sulfoxamines are one candidate to replace neonicotinoids ${ }^{14}$, and have already been widely approved for use. Siviter and colleagues set out to assess the sublethal effects of sulfoxaflor on the agriculturally important pollinator Bombus terrestris. are useful for controlling crop damage caused as learning and foraging ${ }^{4-8}$, affecting nesting
This bumblebee is common in the wild, and is also reared commercially for crop pollination. Although it is convenient to use commercially reared colonies for experiments, the authors chose to use wild colonies - a decision that should be lauded because it enhances the ecological realism of their study.

Siviter et al. collected 332 wild queen bumblebees, assessed them for parasites and used 249 uninfected individuals to start colonies in the laboratory. The authors succeeded in rearing colonies from 52 queens, providing a robust sample size for their experiment. They then randomly allocated pairs of size-matched bee colonies to either control or insecticideexposure groups. The colonies fed at will for two weeks on either sugar water alone or

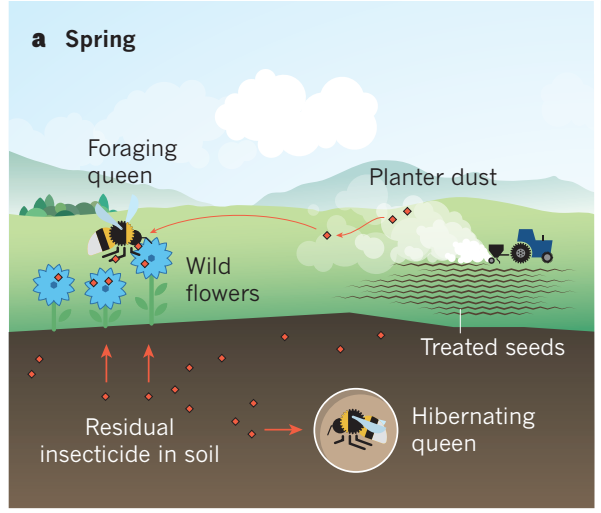

Figure 1 | Routes of bumblebee exposure to insecticides. Siviter $\mathrm{et} \mathrm{al.}{ }^{3}$ have investigated how exposure to the insecticide sulfoxaflor affects bumblebee colonies, using a combined laboratory-field protocol. There are multiple potential routes of exposure to systemic insecticides. a, In spring, insecticide-treated seeds are sown. Contaminated dust from seed planters drifts across fields, and lands on wild flowers (insecticide residues are indicated by red diamonds, routes of spread by red arrows). Residual insecticide in the soil from the previous year might affect queen bumblebees hibernating in the soil, or be taken up by wild flowers, leading to exposure of foraging queens that consume contaminated nectar and pollen. b, In summer, crops grown from treated seeds bloom, producing contaminated nectar and pollen (red stripes). Spray treatments can increase insecticide levels on crops and on nearby wild flowers. Foraging worker bees ingest insecticide-laced nectar and pollen from both treated crops and contaminated wild flowers $^{17,18}$, and are exposed through contact with sprayed plant tissue when foraging on crops. Workers take insecticide-laced pollen and nectar back to the colony, where it is ingested by larvae (not shown). 